\title{
Bicompartmental Decompressive Craniectomy: Report of Two Cases
}

\author{
Saad Akhtar ${ }^{1}$ Badar Ujjan ${ }^{1}$ Muhammad Waqas ${ }^{1} \quad$ Muhammad Waqas Khan $^{1}$ Gohar Javed ${ }^{1}$ \\ ${ }^{1}$ Department of Neurosurgery, Aga Khan University Hospital, Karachi, \\ Pakistan \\ Surg J 2015;1:e41-e43.

\begin{abstract}
Address for correspondence Muhammad Waqas Khan, MBBS, MD, Department of Neurosurgery, Aga Khan University Hospital, National Stadium Road, Karachi, Sindh 74800, Pakistan (e-mail: mwkhan61@gmail.com).
\end{abstract}

\begin{abstract}
Keywords

- bicompartmental decompressive craniectomy

- traumatic brain injury

A recent study of randomized controlled trials showed favorable outcomes with use of decompressive craniectomy in managing and treating uncontrolled intracranial pressures accompanied with cerebral edema due to trauma. We present the details of bicompartmental decompressive craniectomy on two patients who presented with severe head trauma of supra- and infratentorial pathologies. The surgical management techniques and long-term follow-up are discussed in detail.
\end{abstract}

Patients with refractory raised intracranial pressure (ICP) from severe traumatic brain injury remain a challenge to treat despite medical and surgical advances.

In a recent trial, DECRA (decompressive craniectomy in patients with severe traumatic brain injury) has been shown to lower ICP and reduce times on mechanical ventilation in patients who presented with diffuse traumatic brain injury who underwent bifrontal hemicraniectomy. ${ }^{1}$ Similar results have been shown in other clinical trials on decompressive craniectomy. ${ }^{2}$ There is very scarce data available on bicompartmental decompressive craniectomy. A study published by Ecker et al from the Afghanistan and Iraq wars is the only literature that mentions the use of bicompartmental decompressive craniectomy in traumatic brain injury. ${ }^{3}$

\section{Case Reports}

\section{Case 1}

A 32-year-old man presented with severe head injury following an automobile accident (collision of a car with a truck) with a Glasgow Coma Score of 6/15. The patient was intubated and computed tomography (CT) scan was performed, which showed bilateral infratentorial extradural hematoma (EDH) on the left side larger than the right, bifrontal contusion, traumatic subarachnoid hemorrhage, and diffuse brain edema with effacement of basal cisterns (-Fig. 1a). The patient was rushed to the operating room. The options of bilateral fronto-temporo-parietal decompressive craniec-

received

June 22, 2015

accepted after revision

August 27, 2015

published online

October 13, 2015 tomy versus evacuation of posterior fossa extradural and supratentorial decompression were considered. The decision of EDH evacuation followed by supratentorial decompression posed a challenge to positioning during and after the surgery and rehabilitation.

The patient underwent left suboccipital craniectomy and EDH evacuation. After supine repositioning, bifrontal decompressive craniectomies were performed along with expansile duroplasty (-Fig. 1b). The patient was kept in the intensive care unit postoperatively and extubated on postoperative day 2 after his ventilation parameters improved. Follow-up CT scan showed resolving edema ( - Fig. 1c). He was discharged with a Glasgow Outcome Score (GOS) of 4 ( - Fig. 1d and 1e). The patient showed good functional recovery on follow-up.

\section{Case 2}

A 2-year-old boy presented to the emergency room after a motor vehicle accident (car rolled over) with a Glasgow Coma Score of 8/15. He was intubated and a subsequent CT brain revealed a right suboccipital EDH, subdural hematoma, and intraparenchymal hematoma in the right cerebellum. Complete obliteration of basal cisterns along with diffuse brain swelling in the supra- and infratentorial compartments was noted (-Fig. 2a). Right suboccipital decompressive craniectomy and hematoma evacuation along with expansile duroplasty was performed. The decision to perform additional bifrontal decompressive craniectomy was based on diffuse brain swelling in the supratentorial compartment and
Copyright $\odot 2015$ by Thieme Medical Publishers, Inc., 333 Seventh Avenue, New York, NY 10001, USA. Tel: +1(212) 584-4662.
License terms

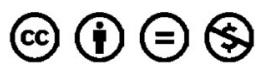

10.1055/s-0035-1565245. ISSN 2378-5128. 

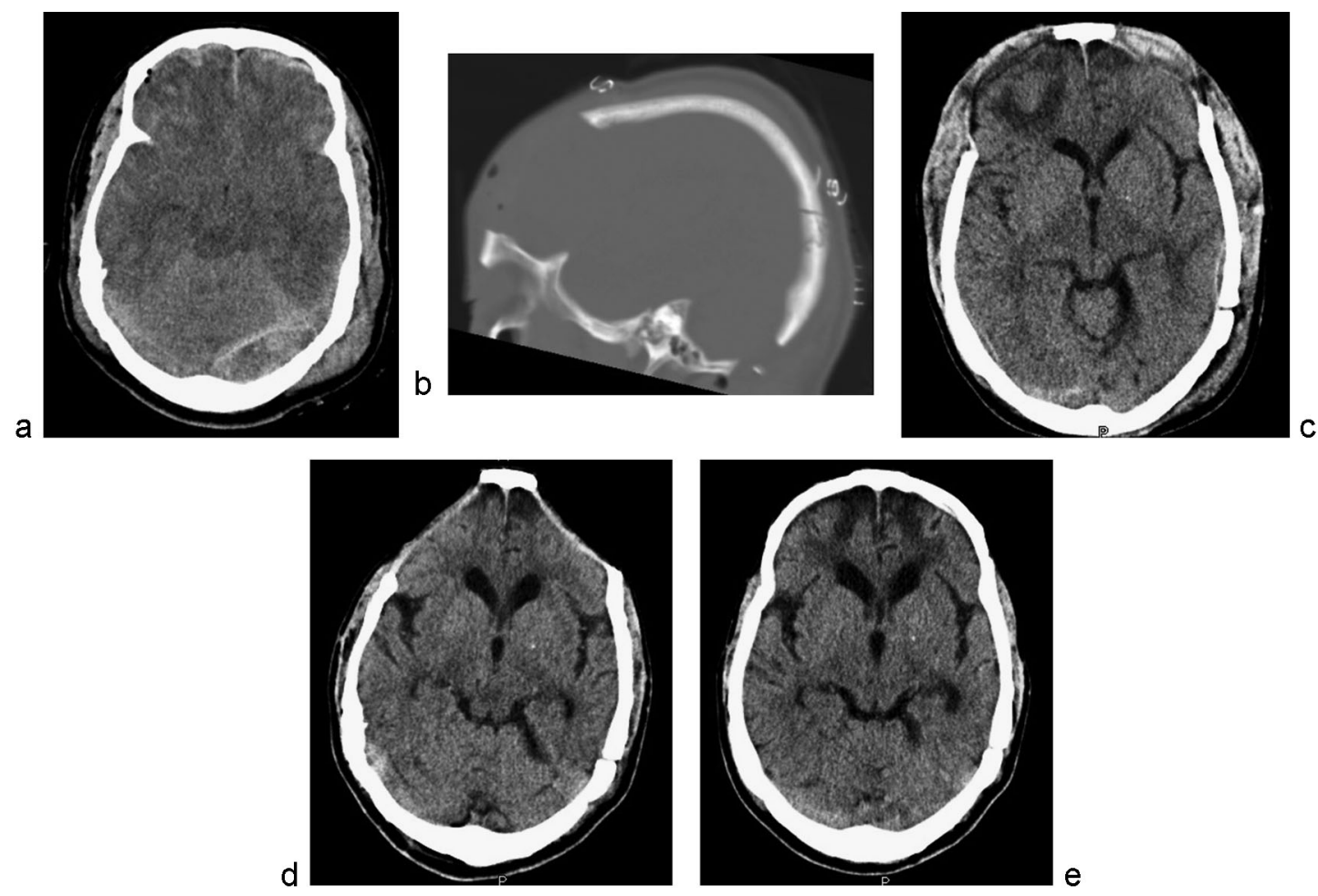

Fig. 1 (a) Preoperative computed tomography (CT) of the brain showing postfossa extradural hematomas, bifrontal contusions, and effacement of basal cisterns. (b) Postoperative sagittal CT (bone window) showing frontal and suboccipital bony defects after bicompartmental decompressive craniectomy. (c) Postoperative CT of the brain showing resolution of brain edema and reappearance of basal cisterns. (d) Delayed postoperative CT scan showing sinking of frontal flaps. (e) CT scan of the brain after autologous cranioplasty.

obliteration of cisterns ( - Fig. 2b). The patient was extubated on the second day and was discharged with a Glasgow Coma Score of 4. Clinical follow-up showed good postoperative recovery ( $\mathbf{- F i g . ~ 2 c ) . ~}$

ICP monitoring was not done in either patient because it is not available in our center.

\section{Discussion}

Decompressive craniectomy is considered as a last resource in the treatment of patients with head trauma with severely raised ICP that is refractory to medical therapy. Unilateral decompressive craniectomy is employed in the case of focal lesions causing a midline shift, and bilateral decompressive craniectomy is used in diffuse head injury or focal lesions involving both hemispheres without a midline shift. ${ }^{4}$ Bicompartmental decompressive craniectomy is indicated when there are separate space-occupying lesions in both the supraand infratentorial compartments leading to isolated raised ICP at each level.

The need for bicompartmental decompressive craniectomy was first felt when the concept of regional brain tissue pressure gradient was studied especially adjacent to the site of a mass lesion. The pressure was highest adjacent to the mass lesion and declined as the distance from the mass lesion increased. Thus, it was concluded that a unicompartmental decompressive craniectomy would not provide adequate decompression for bicompartmental injuries and that a bicompartmental decompressive craniectomy could reduce
ICP more rapidly and effectively than the conventional bifrontal approach. ${ }^{1}$

Bicompartmental decompressive craniectomy therefore can be an effective treatment strategy for uncontrolled ICP in select patients with supratentorial and infratentorial severe traumatic brain injury along with diffuse brain swelling especially if the option of unicompartmental decompression and maximal medical therapy is chosen and continuous ICP monitoring is not available.

In the authors' experience of performing bicompartmental decompressive craniectomy in patients suffering from diffuse brain edema in the supratentorial compartment and mass lesion in the infratentorial compartment, the choice was to perform a bicompartmental decompressive craniectomy instead of bifrontal, unicompartment, or just posterior fossa decompression followed by maximal medical therapy in the absence of availability of continuous ICP monitoring.

Both patients show good outcome (Glasgow Coma Scale $=4$ ) on long-term follow-up. Another fact noted in our case report is the effectiveness in different age group (pediatric and adult).

Bicompartmental decompressive craniectomy was first reported by Ecker et al, who performed this procedure in six soldiers with severe penetrating brain injury from the conflicts in Iraq and Afghanistan. ${ }^{5}$ Although detailed literature was unavailable regarding the pathophysiology of the traumatized brain that led to the use of this technique, we present 2-year follow-up of our patients with excellent outcome. 
a
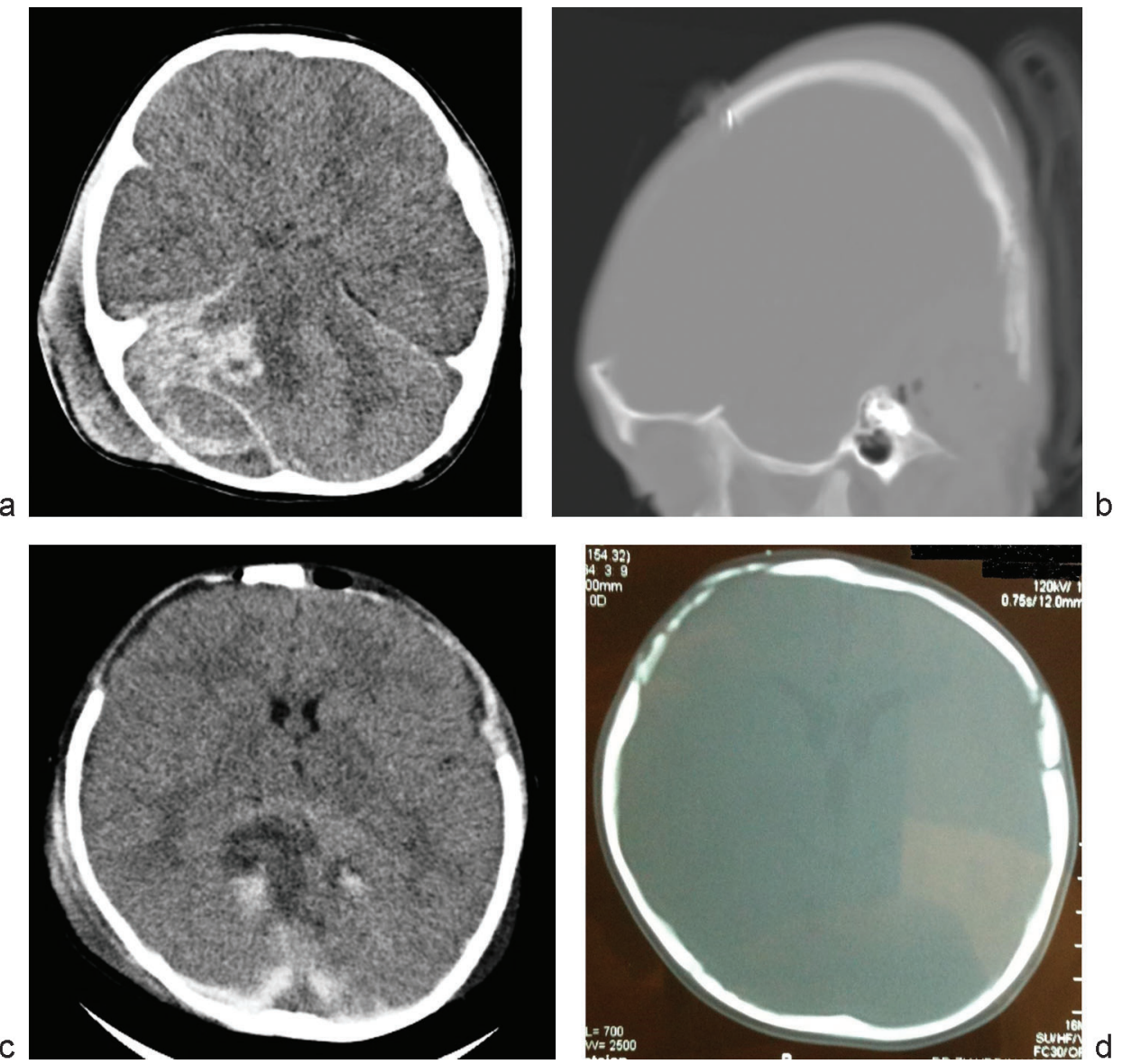

Fig. 2 (a) Preoperative computed tomography (CT) of the brain showing right suboccipital extradural hematoma, subdural hematoma, and intraparenchymal hematoma with generalized brain swelling. (b) Postoperative sagittal CT (bone window) showing frontal and suboccipital bony defects after bicompartmental decompressive craniectomies. (c) Postoperative CT of the brain showing resolution of brain edema and reappearance of basal cisterns. (d) Follow-up axial CT (bone window) at 3 months showing spontaneous bone growth at craniectomy defect.

Nursing care is an important factor when considering the possibility of compression of a herniated brain through the suboccipital craniectomy defect. Stefini et al reported utilizing a foam pillow in which a hole was cut that was slightly larger than the craniectomy. ${ }^{1,3,6}$

\section{Conclusion}

Our case series demonstrates that bicompartmental decompressive craniectomy can be safely performed, with rapid control of ICP in both supra- and infratentorial compartments, and should be considered as an option in patients with bicompartmental head injuries. Larger studies are needed to address clinical indications and long-term outcomes of this technique to replace unicompartmental decompressive craniectomy with bicompartmental decompression in patients with bicompartmental traumatic injury.

\section{References}

1 Cooper DJ, Rosenfeld JV, Murray L, et al; DECRA Trial Investigators; Australian and New Zealand Intensive Care Society Clinical Trials Group. Decompressive craniectomy in diffuse traumatic brain injury. N Engl J Med 2011;364(16):1493-1502

2 Ransohoff J, Benjamin V. Hemicraniectomy in the treatment of acute subdural haematoma. J Neurol Neurosurg Psychiatry 1971; 34(1):106

3 Ecker RD, Mulligan LP, Dirks M, et al. Outcomes of 33 patients from the wars in Iraq and Afghanistan undergoing bilateral or bicompartmental craniectomy. J Neurosurg 2011;115(1):124-129

4 Rinaldi A, Mangiola A, Anile C, Maira G, Amante P, Ferraresi A. Hemodynamic effects of decompressive craniectomy in cold induced brain oedema. Acta Neurochir Suppl (Wien) 1990; 51:394-396

5 Hutchinson PJ, Kirkpatrick PJ. Decompressive craniectomy in head injury. Curr Opin Crit Care 2004;10(2):101-104

6 Stefini R, Bergomi R, Catenacci E, Cereda C, Latronico N, Mortini P. Bi-occipital decompressive craniectomy in refractory post traumatic intracranial hypertension: first report of one case. $\mathrm{Br} \mathrm{J}$ Neurosurg 2007;21(5):527-531 